\title{
A feasibility study on the use of artificial neural networks to model catalytic oxidation in a metallic foam reactor
}

\author{
Mino Woo*, ${ }^{*}$ and Marc E.J. Stettler
}

Department of Civil and Environmental Engineering, Imperial College London, London, SW7 2AZ, UK

\section{S1. Development of 1D Physics Model}

The 1D physics model in the present study has been developed by modifying the verified model for catalytic combustion in monolith channel ${ }^{1}$. The specific surface area of monolithi channel $\left(4 / d_{\mathrm{H}}\right)$ was replaced by the specific surface area of metallic foam structure. Nusselt and Sherwood numbers used for the estimation of interphase transport coefficients were obtained by Eq. (14) corresponding to the structure of a bank of tubes. Instead of the channel diameter, the strut diameter defined by Eq. (15) was employed as the characteristic length. Three major binary reactions in DOC $\left(\mathrm{CO}-\mathrm{O}_{2}, \mathrm{C}_{3} \mathrm{H}_{6}-\mathrm{O}_{2}\right.$ and $\left.\mathrm{NO}-\mathrm{O}_{2}\right)$ were implemented as described in Table 2.

We mainly consulted Koltsakis et al. ${ }^{2-4}$ to establish the basic structure of the model but were unable to generalise the model due to the lack of information provided. For example, the rate of $\mathrm{CO}-\mathrm{O}_{2}$ binary reaction differs depending on the studies ${ }^{2,5,6}$. Their pre-exponential factors differ by several orders of magnitude, and activation energies differ in the range between $95-119 \mathrm{~kJ} / \mathrm{mol}$. Thus, the reaction parameters and inhibition terms were directly fitted to match the measured conversion from our experiments. Figure S1 demonstrates that the model predictions with the fine-tuned reaction parameters are in good agreement with the measured light-off curves. However, the fine-tuned model was only valid in the limited range of test conditions such as having high dependency on inlet compositions. Predictions of largely different sets of test conditions required recalibration of reaction parameters to maintain accuracy.

\footnotetext{
* Corresponding author, Email address: m.woo@imperial.ac.uk

† Present address: National Energy Technology Laboratory, Morgantown, West Virginia 26505, United States
} 


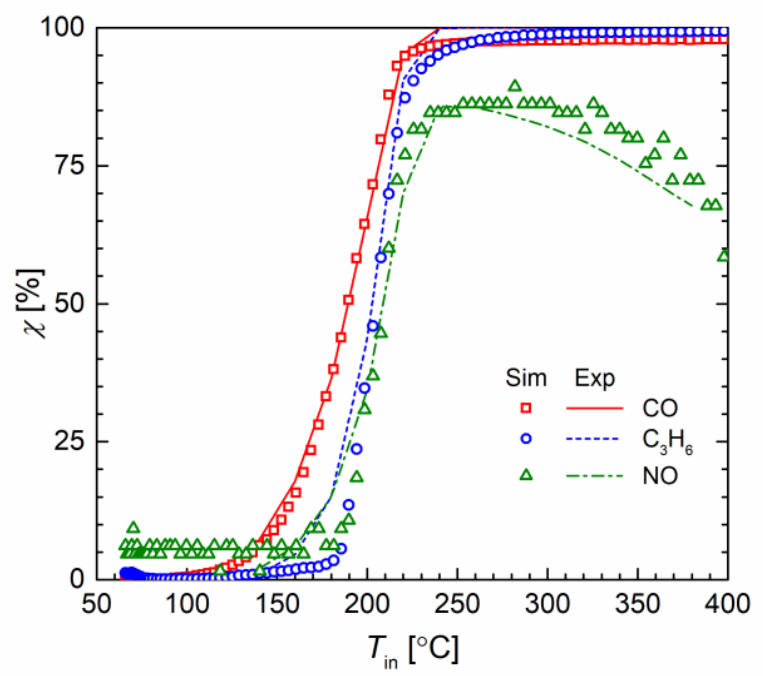

Figure S1: Comparison of predicted (Sim) and measured (Exp) light-off curves for the three major species in the DOC operation in microreactor with metallic foam substrate.

\section{S2. Physics Model with a Threshold Concentration}

In addition to the inhibition term $G$ in Eq. (18), the present physics model employs a threshold concentration to provide a better fit to the measured retardation phenomena with increasing inlet concentration. Figure S2 shows the predicted light-off curves for CO oxidation, with and without the use of the threshold concentration. Without the threshold concentration, the light-off curves were only slightly shifted to the right direction despite the inhibition term $G$ in the rate equations. With the threshold concentration, the predicted $\mathrm{CO}$ conversion were further retarded and were in better agreement with measured value before light-off condition $(<50 \%)$.

While the ad-hoc threshold concentration might be unphysical, it provides for a better fit to the empirical data. The reactions could also be delayed without the threshold concentration by lowering the activation energy and refitting the pre-exponential factor ${ }^{7}$. However, this also resulted in poorer match with regards to the increasing SV. Aside from this, additional surface coverage parameter could be used to fit such phenomena, which introduces an additional fitting parameter ${ }^{8}$ :

$$
R_{\mathrm{S}}=\frac{A \exp \left(-E_{\mathrm{A}} / R T_{\mathrm{S}}\right)}{G} x_{\mathrm{CO}} \exp \left(\frac{-\varepsilon x_{\mathrm{CO}}}{R T_{\mathrm{S}}}\right)
$$




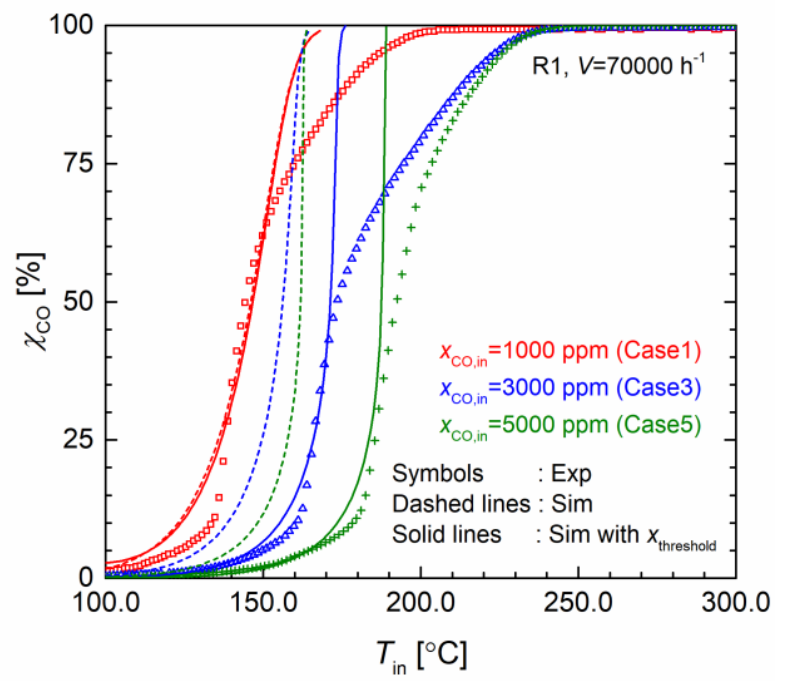

Figure S2: Comparison of predicted light-off curves for CO oxidation without threshold concentration (dashed line) and with threshold concentration (solid line) against the measured values (symbols).

where $\varepsilon$ is a parameter for surface species which serves as another fitting parameter. This model provides similar results as for the present model with the threshold concentration. Although this approach seems to be more physical, it is more complicated to find the fitting parameters than the use of threshold concentration. Moreover, the inhibition term $G$ was also modified in those two previous models, which might lead it to being less applicable for the practical condition such as synthetic gas.

The concept of threshold concentration in this study was inspired by the measured behaviours in the species consumption. Figure S3 shows the measured consumption rates of the major three species with various inlet concentrations. The slopes of $\mathrm{CO}$ and $\mathrm{C}_{3} \mathrm{H}_{6}$ conversion curves in Figure S3 (a) and (b) are generally proportional to the inverted temperature though $\mathrm{C}_{3} \mathrm{H}_{6}$ conversion curves are not absolutely monotonic, while the NO curves shown in Figure S3 (c) are clearly non-monotonic depending on the balance of oxidation and reduction as a function of temperature. Moreover, the slopes of $\mathrm{CO}$ conversions for $2.2 \times 10^{-3}<1 / T<3 \times 10^{-3}$ are almost constant regardless of the inlet composition. This indicates that the consumption rate in that temperature range is independent of the inlet composition, which is likely due to the limit of active sites on the catalyst surface. 

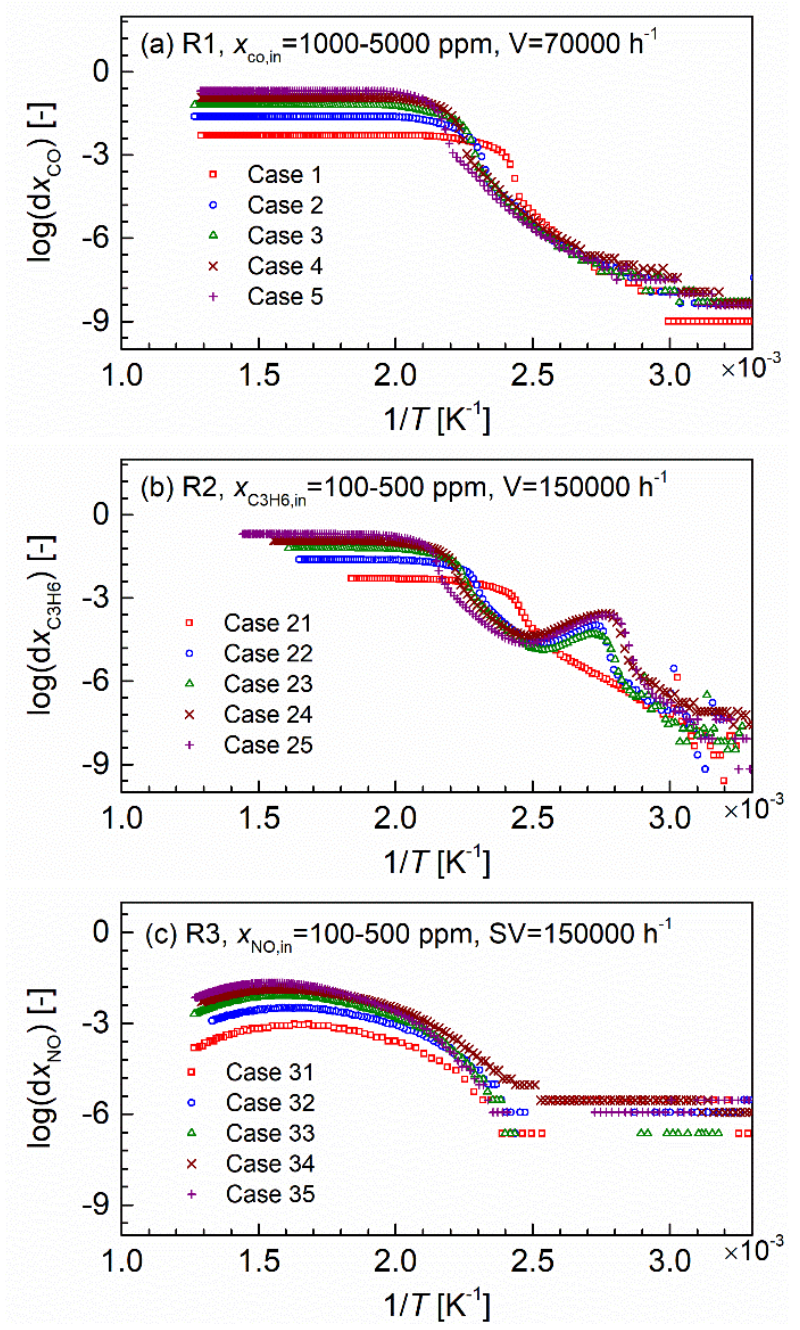

Figure S3: The measured species consumption rates of (a) $\mathrm{CO}$, (b) $\mathrm{C}_{3} H_{6}$ and (c) $\mathrm{NO}$ as a function of inverted temperature.

\section{S3. Parametric Study of Heat and Mass Transfer Coefficients}

The mass transfer coefficient that determines the amount of mass transferred between the gas and solid phases is one of the key parameters governing the species conversion in the heterogeneous reaction model. This section presents the parametric study of heat and mass transfer coefficients affecting the light-off curves for CO oxidation. Figure S4 (a) shows the CO light-off curves for the inlet concentration of 1000 ppm and $5000 \mathrm{ppm}$ depending on the temperature. Multiplication factors (f) with various orders of magnitude were used to scale the heat and mass transfer coefficients. There were only slight changes for the f of 10, 1 , and 0.1 . For $\mathrm{f}=0.01$, the slope of the curve after the light-off condition $(>50 \%)$ became gentler. For 
$\mathrm{f}=0.001$, the conversion has reached a plateau of approximately $25 \%$ of conversion despite further increasing temperature. The delay observed after the light-off condition is a known phenomenon with the system that is mass transfer limited.

Figure S4 (b) shows the ratio between the mass transfer rate and the reaction rate at the inlet depending on the temperature. The mass transfer rate for the original system (solid line) is always higher than the reaction rate, and, only with $\mathrm{f}=0.001$, it approaches to parity. This represents that the system is not mass transfer limited in the given test conditions. It is also noted that, the trends in the ratio differ depending on the inlet concentration. Even with the lowered mass transfer coefficient, the slope of the light-off curve for 5000 ppm is still steep. Moreover, the decrease in mass transfer coefficient resulted in the reduction of the gap between the two light-off curves for $1000 \mathrm{ppm}$ and $5000 \mathrm{ppm}$. This indicates that the retardation of conversion by increasing inlet concentration is not significantly dictated by the mass transfer coefficient, but it might be more affected by the inhibition effect among the surface species competing for limited surface sites for reactions. This evidence supports the fact that, with onestep global reaction mechanisms that include only one rate-limiting step in the form of rate equation, the conversion depends more strongly on the inhibition term (or empirical parameters needed to fit such retardation phenomena) than the mass transfer coefficient.

(a)

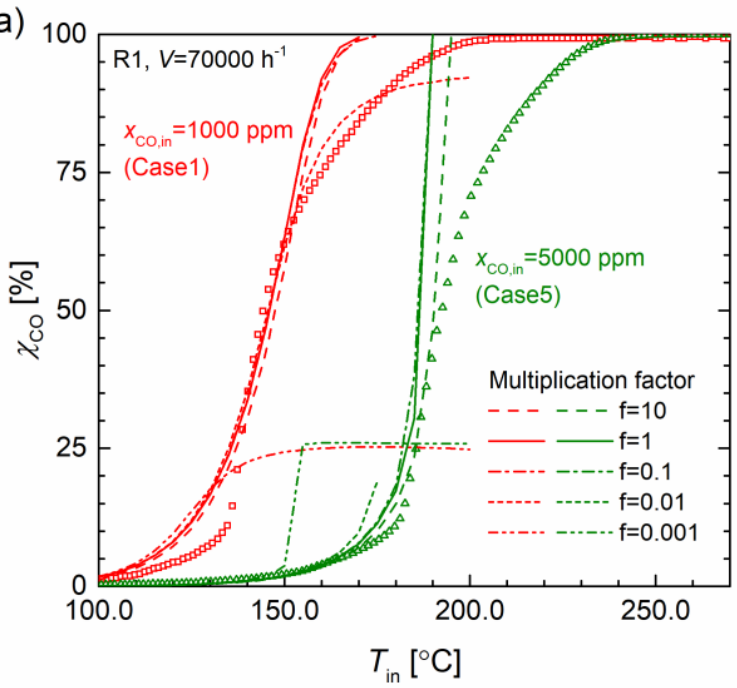

(b)

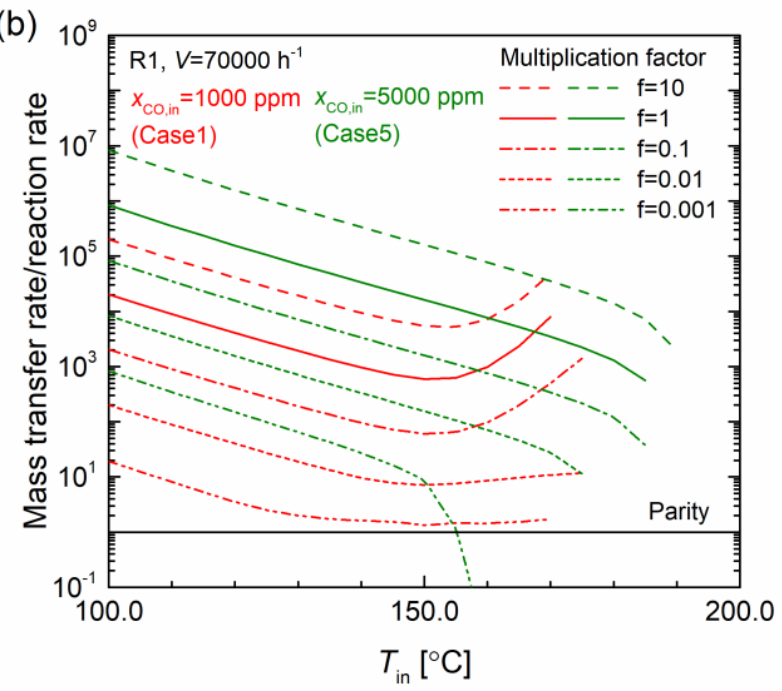

Figure S4: The results of parametric study of the mass (and heat) transfer coefficients multiplied by a factor with different orders of magnitude. (a) CO light-off curve and depending on the mass (and heat) transfer coefficient, and (b) the ratio between mass transfer rate and reaction rate at the inlet. 


\section{References}

1. Hayes, R. E.; Kolaczkowski, S. T., Introduction to Catalytic Combustion. Taylor \& Francis: 1998.

2. Koltsakis, G. C.; Katsaounis, D. K.; Samaras, Z. C.; Naumann, D.; Saberi, S.; Böhm, A.; Markomanolakis, I., Development of Metal Foam Based Aftertreatment System on a Diesel Passenger Car. In SAE International: 2008.

3. Koltsakis, G. C.; Katsaounis, D. K.; Markomanolakis, I. A.; Samaras, Z. C.; Naumann, D.; Saberi, S.; Böhm, A., Design and Application of Catalyzed Metal Foam Particulate Filters. In SAE International: 2006.

4. Koltsakis, G. C.; Katsaounis, D. K.; Markomanolakis, I. A.; Samaras, Z. C.; Naumann, D.; Saberi, S.; Böhm, A., Metal Foam Substrate for DOC and DPF Applications. In SAE International: 2007.

5. Matthess, N.; Schweich, D.; Martin, B.; Castagna, F., From Light-Off Curves to Kinetic Rate Expressions for Three-Way Catalysts. Topics in Catalysis 2001, 16, (1), 119-124.

6. Triana, A. P.; Johnson, J. H.; Yang, S. L.; Baumgard, K. J., An Experimental and Numerical Study of the Performance Characteristics of the Diesel Oxidation Catalyst in a Continuously Regenerating Particulate Filter. In SAE International: 2003.

7. Woo, M.; Kim, K.; Kim, H.; Lee, J.; Lee, B. In Kinetic Study of Catalytic Reactions in the Metalfoam based DOC using Micro-Reactor System, The Korean Society of Automotive Engineers, 2009; 2009.

8. Woo, M.; Kim, K.; Lee, J. In Numerical modeling for catalytic oxidation characteristics of metal foam after-treatment systems by using 1D PFR model, The Korean Society of Automotive Engineers, 2010; 2010. 